\title{
Morphological alteration in mitochondria following diclofenac and ibuprofen administration.
}

\begin{abstract}
This study was conducted to identify and to compare the mitochondrial morphological alterations in livers of rats treated with various doses of diclofenac and ibuprofen. Hundred and forty-four male Sprague Dawley rats were dosed with 3, 5 and $10 \mathrm{mg} \mathrm{kg}-1$ diclofenac and ibuprofen in saline via intraperitoneal injection for 15 days. The control group was administered with saline in a similar manner. Four rats were euthanised every 3 days until day 15 . While $200 \mathrm{mg} \mathrm{kg-1}$ diclofenac and ibuprofen-treated rats $(n=4)$ were euthanized 10 $\mathrm{h}$ post-treatment. The livers were removed, cleaned and a section across the right lobe was taken and fixed in 4\% (v/v) glutaraldehyde for electron microscopy analysis and the remaining samples were kept at $-80^{\circ} \mathrm{C}$ for Western blot analysis. Five milligram per kilogram and $10 \mathrm{mg} \mathrm{kg}-1$ diclofenac-administered rats for 15 days revealed the presence of enlarged mitochondria, irregular and ruptured mitochondrial membranes. While rats administered with $10 \mathrm{mg} \mathrm{kg}-1$ ibuprofen also showed the presence of mitochondria with irregular membrane structure and ruptured membranes. Western blotting analysis of mitochondrial fractions revealed the expression of cytochrome $\mathrm{c}$ in all samples and complete absence of cytochrome c expression in the cytosolic fraction of all samples after day 15. Analysis in $200 \mathrm{mg} \mathrm{kg}-1$ diclofenac and ibuprofen-treated groups, revealed expression of cytochrome $\mathrm{c}$ in both mitochondrial and cytosolic fractions. This observation indicates that both diclofenac and ibuprofen may alter the morphology of mitochondria, leading to cytochrome $\mathrm{c}$ release into the cytosol. Further studies needs to be conducted to investigate on the activity of the mitochondria following both treatments.
\end{abstract}

Keyword: Cytochrome c; Hepatotoxicity; Mitochondria; Non-steroidal anti-inflammatory drugs. 